\title{
In recognition of aberration-corrected TEM
}

\author{
The efforts to develop electron lens systems that can achieve atomic resolution in transmission electron \\ microscopy have been awarded the most prestigious accolade dedicated to nanoscience.
}

T he 2020 Kavli Prize in nanoscience was awarded to four scientists for their contribution to the field of aberration correction in transmission electron microscopy (TEM). Harald Rose, Maximilan Haider, Knut Urban and colleagues published the first aberration-corrected conventional TEM images in $1998^{1}$. Ondrej Krivanek and colleagues published aberration corrected scanning TEM images with sub-ångström resolution in $2002^{2}$.

News of the award should be celebrated for a number of reasons. First and foremost, because it recognizes the scientific possibilities that the efforts of these scientists have allowed. Transmission electron microscopy allows looking inside the structure of materials with a much higher resolution than optical microscopy. But it is only with aberration correction that we can peer deep into matter and connect the properties of a material with those of single atoms. Microscopists can now determine the position of each of these single atoms with the precision of a few picometres. This ability has been used, for example, to explore the origin of ferroelectric domains with a conventional aberration-corrected TEM by looking purely at the minimal atomic displacements in the material lattice ${ }^{3}$. Furthermore, when used in a scanning TEM, aberration correction allows focussing a beam of electrons very precisely on a single atomic column in a lattice, and performing what is known as electron energy-loss spectroscopy. With this technique, scientists are able to investigate the chemical nature of the atoms in a material and even the type of bonding types in a lattice ${ }^{4}$.

This year's prize honours also the history of the field and the determination of theoretical and experimental physicists to achieve a precise goal: improving the resolution of transmission electron microscopes to obtain atomic resolution. It is a quest that has lasted around six decades. Already in the mid-1930s, shortly after the invention of the TEM by Ernst Ruska, physicists such as Otto Scherzer had started investigating ways to correct electronic lenses from naturally occurring aberrations. But we had to wait until 1990,

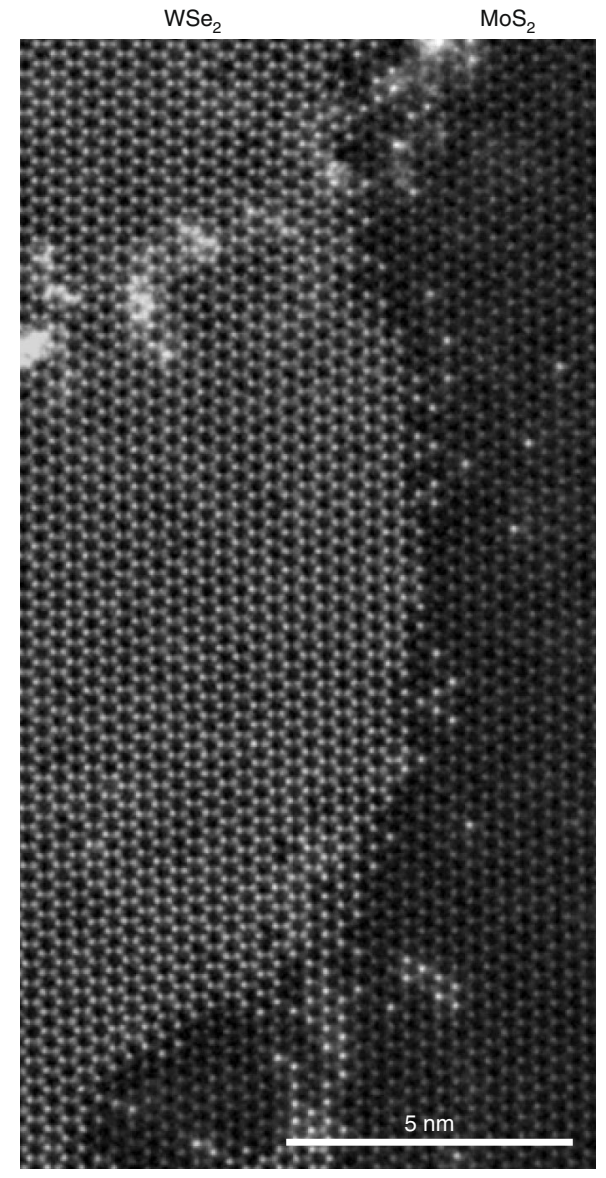

Fig. 1 | Atomic resolution images of two-dimensional materials. Annular dark field scanning TEM image of a lateral heterojunction between $\mathrm{WSe}_{2}$ and $\mathrm{MoS}_{2}$. Adapted with permission from ref. 7 , Springer Nature Ltd.

when a feasible design was published by Harald Rose ${ }^{5}$. A design that he would then realize experimentally with Haider and Urban in the late 90s, a few years before the sub-ångström resolution demonstrated by Krivanek with a scanning TEM.

Perhaps more subtle, a reason for celebration should also be the decision of the Nanoscience Kavli Committee to put fairness before tradition, thus awarding the prize to four scientists for the first time since the prize was first awarded in 2008. Rigorously restricting the maximum number of laureates, typically to three, can be a serious limitation. Too many times has this raised eyebrows, in connection with the Nobel Prize for example, arguably the most prestigious scientific award. And it is not too far-fetched to imagine that some scientific discoveries have not been recognized with the Nobel Prize because it was impossible to restrict the list of laureates to three. As mentioned, the Kavli Prize is also traditionally awarded to a maximum of three scientists. However, the committee has the faculty to extend the number if necessary. In this case, after scrupulous research it was decided that all these four scientists deserved recognition.

Finally, we must recognize what aberration-corrected TEM allows materials scientists and nanotechnologists to do today. The properties of very sharp interfaces exhibiting emerging phenomena can be studied in fine detail. Furthermore, the continuous developments in both electron lenses and electron detectors has permitted resolving atoms of lighter elements such as carbon, allowing the investigation of the relation between structure and properties of carbon nanotubes, graphene and other two-dimensional materials (Fig. 1).

To recognize the work of this year's Kavli Prizes, in astrophysics, nanoscience and neuroscience, editors of Nature journals have prepared an online collection, gathering publications from the laureates and papers from other scientists that represent important milestones ${ }^{6}$. We invite you to browse through the collection and to join us in congratulate this year's Kavli laureates for their extraordinary achievements.

Published online: 10 June 2020

https://doi.org/10.1038/s41565-020-0721-6

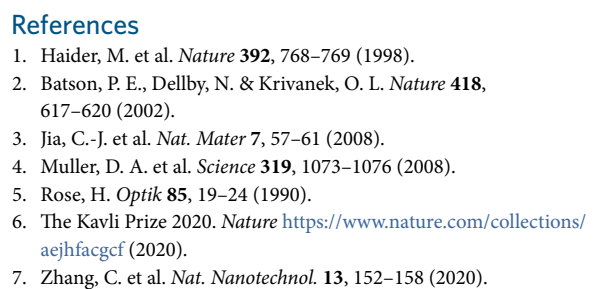

